Original Article

Journal of Epilepsy Research pISSN 2233-6249 / elSSN 2233-6257

Received October 29, 2020

Revised May 5, 2021

Accepted May 9, 2021

Corresponding author:

Sweta Kumari, MD, DNB

Department of Pediatrics, ESI PGIMSR,

Basaidarapur, New Delhi 110015, India

Tel. +91-9990407599

Fax. +91-9990407599

E-mail; sweta.csmmu@gmail.com

\section{Electroencephalogram versus Magnetic Resonance Imaging Brain as the Initial Investigation of Choice in Neurologically Normal Children with First Afebrile Seizure in India}

\author{
Jyoti Bagla, MD , Harpreet Kaur, DCH ${ }^{1}$, Anu Singhal, DNB ${ }^{2}$, Devendra Mishra, MD², \\ Sweta Kumari, MD, DNB ${ }^{1}$, Anand Prakash Dubey, MD', Sandhya Soneja, DNB ${ }^{1}$ \\ Departments of ${ }^{1}$ Pediatrics and ${ }^{2}$ Radiodiagnosis, ESI PGIMSR, New Delhi; ${ }^{3}$ Department of Pediatrics, Maulana \\ Azad Medical College, New Delhi, India
}
Background and Purpose: To compare the rates of clinically relevant information provided by electroencephalogram (EEG) and magnetic resonance imaging (MRI) brain in first afebrile seizure (FAS) in children.

Methods: In this prospective randomized controlled trial, neurologically normal children between the age of 2 and 14 years, presenting with first episode of unprovoked, afebrile generalized or partial seizures, were included. Enrolled patients were randomized into two groups. After stabilization, initial workup and management, group I-patients underwent an EEG followed by MRI, whereas group II-patients underwent an initial MRI brain followed by an EEG. The patients were followed up after results of both the investigations and then every 3 months for seizure recurrence. The primary outcome was the proportion of investigations, providing clinically relevant information. The secondary outcomes were to determine the etiological diagnosis of FAS and record adverse events associated with EEG and MRI.

Results: Out of 170 enrolled patients, 52 patients (61.2\%) in initial EEG group and 53 patients (70.6\%) in initial MRI group had abnormal results on first investigation. An etiological diagnosis could not be made in any patient in initial EEG group. Neuroimaging revealed an etiological diagnosis in 53 patients (70.6\%) in initial MRI group. Inflammatory granuloma was found to be the most common cause of FAS, followed by idiopathic epilepsy.

Conclusions: The results of our study done in neurologically normal children with FAS showed a high diagnostic yield with an initial MRI. We recommend MRI brain to be considered as the initial investigation for evaluation of FAS in children. (2021;11:56-62)

Key words: First afebrile seizure, Inflammatory granuloma, Magnetic resonance imaging brain, Electroencephalography, Epilepsy

\section{Introduction}

Seizures are the commonly seen pediatric neurological disorders, affecting approximately $10 \%$ of pediatric population, which contribute to $5 \%$ of all medical visits to emergency department. ${ }^{1}$ However, seizures occur more commonly in the developing countries like India, which accounts for $80 \%$ of burden of the disease. Increased prevalence and incidence in these areas may be related to factors such as low socioeconomic status, limited access to health care and higher frequency of intracranial infections like neurocysticercosis (NCC) and tuberculosis. ${ }^{2}$ The overall prevalence of epilepsy in India is 5.59 per 1,000 population, ${ }^{3}$ with 1.1 per thousand in $2-<6$ years and 2.2 per thousand in 6-9 years. ${ }^{4}$ Depending upon the hospital policy and the clinician's choice, about $70 \%$ of these children are managed as inpatients. ${ }^{5} \mathrm{~A}$ first episode of seizure poses challenge for the pediatrician to plan the management options and counsel family for short-term and long-term outcomes. Overall prognosis of seizure with respect to seizure control and recurrence depends upon the underlying etiology which may range from a benign reversible encephalopathy or an epileptogenic brain foci. ${ }^{6}$ In a case of first afebrile 
seizure (FAS), diagnostic evaluation influences therapeutic options including need of hospital admission, counselling of the family members, and specific follow-up plans. In India the common etiologies of FAS are single small contrast enhancing computed tomography lesion (SSECTL) (NCC and tuberculomas). Infarct, tumor and miscellaneous causes like periventricular leukomalacia, hydrocephalus, cerebral atrophy and etc. are other less common causes of FAS.'

The practice parameter of American Academy of Neurology (AAN) for evaluation of FAS in children recommends electroencephalogram (EEG) as an essential investigation for all children. ${ }^{8}$ Indian Academy of Pediatrics also recommends EEG as a part of initial evaluation in all children presenting with an epileptic event. ${ }^{9}$ Guidelines for Epilepsy Management in India states that ideally 'all' children with epileptic seizures should have an EEG and 'most' children with epilepsy should have an elective magnetic resonance imaging (MRI), ${ }_{1}^{10}$ whereas ILAE suggests that EEG is 'essential' only for those younger than 2 years of age. ${ }^{11}$ All these guidelines are more than a decade old and have not been revised in recent times. The AAN guideline reports about insufficient evidence to support a recommendation for the neuroimaging performed for the sole indication of having a history of seizure after a FAS in children. However, they do recommend that MRI is the preferred neuroimaging modality when it is considered and provide certain conditions where MRI should be seriously considered. ${ }^{8}$ Whereas Indian authors recommend EEG as a mandatory investigation for those having unprovoked seizure with normal neuroimaging findings. ${ }^{12}$ However, neuroimaging is preferred as first investigation prior to EEG for Indian children with unprovoked seizure due to the higher incidence of focal structural lesions like NCC or tuberculoma. ${ }^{13}$ American and European guidelines differ from the Indian approach, preferring EEG as the first-line investigation for cases of unprovoked seizure. ${ }^{8}$ We conducted this study to compare the rates of clinically relevant information/diagnostic information provided by EEG and MRI in FAS in children, so as the sequence and need of both these investigations may be evaluated.

\section{Methods}

This was a prospective randomized controlled trial conducted at the department of pediatrics in a tertiary care referral hospital catering to insured population in India. The study was approved by the Institutional Ethics Committee and registered at Clinical Trials Registry - India with registration number REF/2012/12/004374. Neurologically normal children between the age of 2 and 14 years, presenting with first episode of unprovoked, afebrile generalized or partial seizures (or suspected seizures), were included. Exclusion criteria were children with a history of developmental delay, abnormal head circumference, abnormal neurological examination and those with status epilepticus, seizures associated with an acute central nervous system infection (e.g., meningitis, encephalitis), head injury, systemic illness known to be associated with seizures (e.g., Shigella encephalopathy, subacute sclerosing panencephalitis), or recognizable dysmorphism or neurocutaneous syndrome. Patients admitted for suspicion of seizure but discharged with an alternative diagnosis (e.g., breath-holding spell). Pseudoseizure was also excluded from the study.

The following definitions were used for diagnosis and etiologic evaluations of the seizures: 1) first unprovoked afebrile seizure (FUS): one or multiple seizures within a 24-hour period with recovery of consciousness between episodes; 2 ) acute symptomatic: seizure in a previously neurologically normal child, within a week of an underlying etiology including central nervous system infection, encephalopathy, head trauma, cerebrovascular disease, and metabolic or toxic derangements; 3) remote symptomatic: seizure in the absence of an identified acute insult but with a history of a pre-existing neurological abnormality more than 1 week before; and 4) idiopathic epilepsy: seizure that is not symptomatic and occurred in a child with no prior neurological disorder or in a child in whom no neurological findings detected via physical examination. Idiopathic generalized epilepsy specifically refers to the epilepsy syndromes: juvenile myoclonic epilepsy, juvenile absence epilepsy, childhood absence epilepsy, and generalized tonic-clonic seizures alone.

Enrolled patients were randomized using computer-generated random numbers into two groups by a person, not directly involved in the conduct of the study. The random sequence was concealed using opaque labeled envelopes and opened at the time of allocation. After stabilization, initial workup and management, all the patients were subjected to both EEG and MRI in a sequential manner as per their group. Group I-patients first underwent an EEG followed by an MRI, whereas group II-patients underwent an initial MRI brain followed by an EEG. The initial workup also included hematological tests (complete blood count), biochemical tests (blood glucose, serum calcium, serum electrolytes $(\mathrm{Na}, \mathrm{K})$, blood urea and serum creatinine. Lumbar puncture and tuberculin skin test were done depending on the clinical status. 


\section{EEG protocol}

The EEGs were performed using the standard 10-20 international system. All EEG recordings were done for 30 minutes duration with hyperventilation and intermittent photic stimulation at 1-30 Hz. Sleep deprived EEG with sleep deprivation of at least 4-6 hours and simultaneous video recordings of EEG were obtained when required. In patients who required sedation, triclofos (20 mg/ $\mathrm{kg} / \mathrm{dose}$ ) was used. The records were saved in compact discs labeled with patient's identification number. All EEGs were reported by a single, qualified pediatric neurologist, without access to the MRI findings, while blinded to patient identity.

\section{MRI protocol}

The standard MRI sequence employed for evaluation included sagittal and axial T1, axial spin-echo proton density and T2, coronal T2, and high-resolution coronal $\mathrm{T} 2$ of the mesial temporal lobe structures. Brain MRI scans were routinely prescreened by the attending radiologist and contrast administered when indicated (e.g., mass, inflammation). MRI findings were reported by a single, qualified radiologist, without access to the EEG findings, while blinded to patient identity. All participants were followed up after results of both the investigations were available, which was within 1-2 weeks for all. The final diagnosis was made after review of all the investigations. Families not returning for follow-up within 3 days of their scheduled appointment were contacted telephonically and the follow-up visit was rescheduled. The patients were subsequently followed up every 3 months for any seizure recurrence to ensure compliance to antiepileptic drugs if prescribed and monitor the adverse effects of prescribed antiepileptic drugs. The primary outcome was the proportion of investigations providing clinically relevant information, which alters or influences management. The secondary outcomes were to determine the etiological diagnosis of FAS in the study group, and record adverse events associated with EEG and MRI in children with FAS.

\section{Sample size}

Based on the results of a previous study, where show that the proportion of children who had any 'clinically relevant information' with an initial EEG was $20 \%{ }^{7}$ and $5 \%{ }^{8}$ with an initial MRI, 76 patients in each group were required to show significant difference at a two-tailed alpha error of 0.05 and $80 \%$ power. Expecting a drop-out

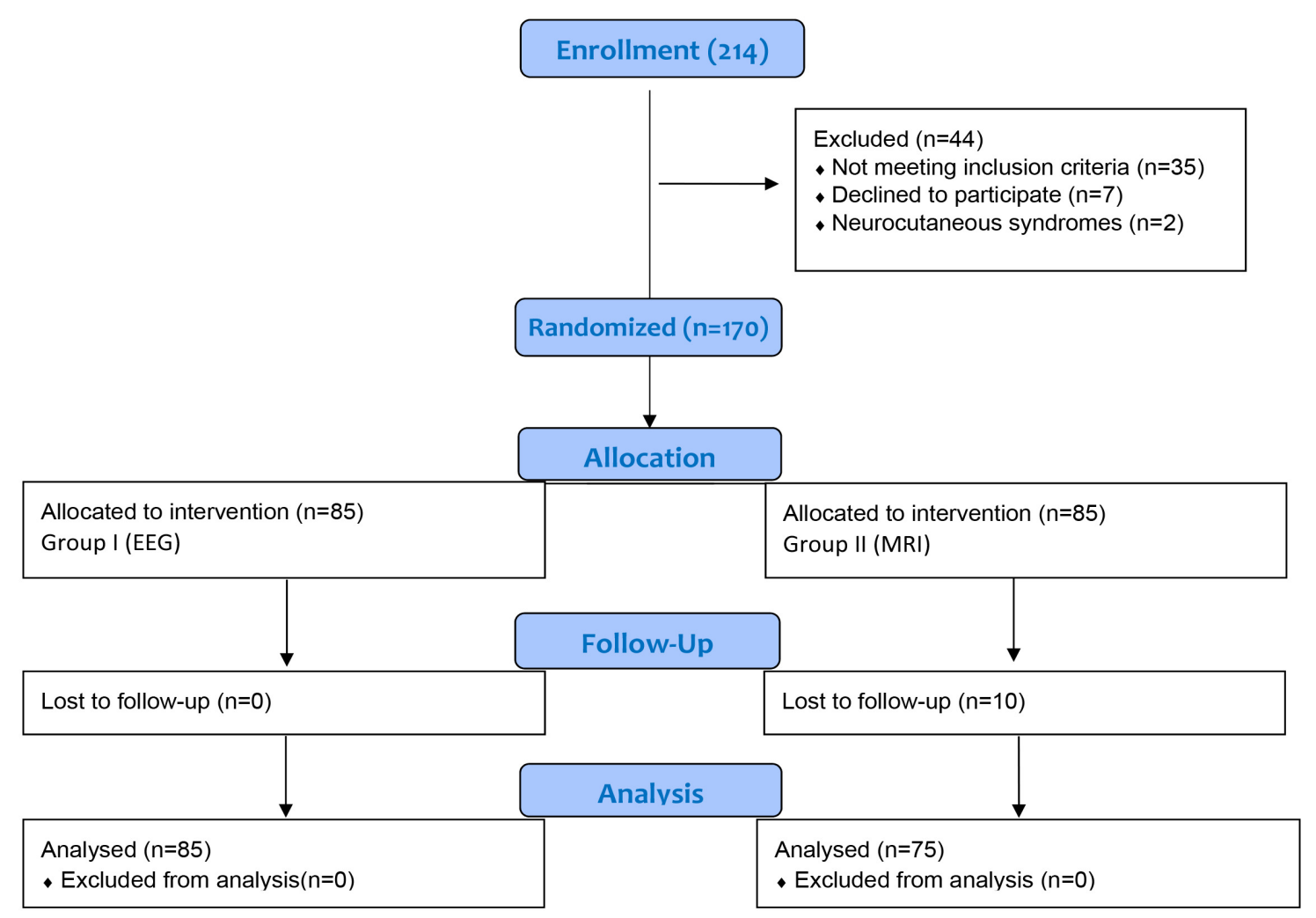

Figure 1. Flow diagram of the study. EEG, electroencephalogram; MRI, magnetic resonance imaging. 
rate of around $10 \%$, it was aimed to enroll 85 patients per group (total subjects, 170).

\section{Statistical analysis}

Demographic and clinical details of all patients were entered in a pre-tested structured proforma. The collected data was entered in MS excel sheet and checked manually for consistency and missing values. The data was statistically analyzed by the statistician using SPSS ver. 21.0 software (IBM SPSS, Inc, Chicago, IL, USA). Chi square/Fisher exact test was used for categorical variables as appropriate. Normally distributed continuous variables were compared by Student $t$-test and continuous variables with skewed distribution were compared by Wilcoxson rank sum Test. A $p$-value of less than 0.05 was considered significant.

\section{Results}

A total of 214 patients were screened for eligibility; 170 fulfilled the eligibility criteria and were enrolled. Since 10 patients were lost to follow up, 160 patients (85 in group I and 75 in group II) were analyzed (Fig. 1). The two groups were similar in the baseline characteristics (Table 1). On analysis, 52 patients (61.2\%) in group I and 53 patients $(70.6 \%)$ in group II had abnormal results on first investigation $(p=0.244)$. An etiological diagnosis could not be made in any patient in group I with the initial EEG. Neuroimaging revealed an etiological diagnosis in 53 patients (70.6\%) in group II (Table 2). On further analysis, 25 out of 160 patients (15.6\%) had an abnormal EEG and gave an etiological diagnosis of idiopathic epilepsy and temporal lobe epilepsy, whereas 111 out of 160 patients (69.3\%) revealed an etiological diagnosis with an abnormal MRI.

Further, an etiological diagnosis was made in 58 (inflammatory granuloma, remote symptomatic and structural malformation) out of 85 patients in group I by MRI brain performed subsequently, whereas

Table 1. Baseline patient and disease characteristics of the study population $(n=160)$

\begin{tabular}{lccc}
\hline & Initial EEG group $(\mathrm{n}=85)$ & Initial MRI group $(\mathrm{n}=75)$ & $p$-value \\
\hline Age (years) & $8(6,9)$ & $8(6,10)$ & 0.245 \\
Weight $(\mathrm{kg})$ & $20(15.5,23.5)$ & $20.0(17.0,27.0)$ & 0.501 \\
Height $(\mathrm{cm})$ & $122.0(112.0,131.0)$ & $123.0(112.0,133.0)$ & 0.751 \\
Head circumference $(\mathrm{cm})^{*}$ & $46.5(43.5,49.0)$ & $46.5(44.7,48.2)$ & 1.000 \\
Male & $46(54.1)$ & $42(56.0)$ & 0.874 \\
Family history of seizure & $13(15.3)$ & $7(9.3)$ & 0.339 \\
Seizure duration, $>5$ minutes & $71(83.5)$ & $58(77.3)$ & 0.423 \\
Further seizure during hospital stay & $3(3.5)$ & $5(6.7)$ & 0.476 \\
History of NICU admission & $2(2.3)$ & $2(2.6)$ & 1.000 \\
Generalized Seizure & $48(56.5)$ & $48(64.0)$ & 0.623 \\
Focal seizure & $37(43.5)$ & $27(36.0)$ & 0.332 \\
\hline
\end{tabular}

Values are presented as median (interquartile range) or number (\%).

$\mathrm{EEG}$, electroencephalogram; $\mathrm{MRI}$, magnetic resonance imaging; $\mathrm{NICU}$, neonatal intensive care unit.

*For age group less than 5 years, initial EEG group=12, initial MRI group $=10$.

Table 2. Results of investigations and etiological diagnosis in children with first seizure $(n=160)$

\begin{tabular}{lccc}
\hline & Initial EEG group $(\mathrm{n}=85)$ & Initial MRI group $(\mathrm{n}=75)$ & $p$-value \\
\hline Abnormal test result on first investigation & $52(61.2)$ & $53(70.6)$ & 0.244 \\
Etiological diagnosis with initial investigation & $0(0.0)$ & $53(70.6)$ & 0.000 \\
Time taken for investigation (days) & $3(2,5)$ & $10(4,22)$ & $<0.001$ \\
Hospital visits* & $3(1,3)$ & $3(2,3)$ & 0.001 \\
\hline
\end{tabular}

Values are presented as median (interquartile range) or number (\%).

$\mathrm{EEG}$, electroencephalogram; MRI, magnetic resonance imaging.

*No of hospital visits, other than the emergency visit, for getting the study investigation done. 
Table 3. Distribution of etiologic diagnosis across the study groups $(n=160)$

\begin{tabular}{lccc}
\hline & Initial EEG group $(\mathrm{n}=85)$ & Initial MRI group $(\mathrm{n}=75)$ & $p$-value \\
\hline Inflammatory granuloma & $51(60.0)$ & $43(57.3)$ & 0.732 \\
Remote symptomatic & $6(7.1)$ & $8(10.7)$ & 0.420 \\
Structural malformation & $1(1.2)$ & $2(2.7)$ & 0.488 \\
Idiopathic epilepsy & $13(15.3)$ & $11(14.7)$ & 0.911 \\
Epilepsy syndrome (temporal lobe epilepsy) & $0(0.0)$ & $1(1.3)$ & 0.285 \\
FUS & $14(16.5)$ & $10(13.3)$ & 0.545 \\
\hline
\end{tabular}

Values are presented as number (\%).

EEG, electroencephalogram; MRI, magnetic resonance imaging; FUS, first unprovoked afebrile seizure.

Table 4. Distribution of etiologic diagnosis in the combined study

\begin{tabular}{lcccc}
\hline & Total $(n=160)$ & Generalized seizure $(n=96 ; 60 \%)$ & Focal seizure $(n=64 ; 40 \%)$ & $p$-value \\
\hline Inflammatory granuloma & $94(58.75)$ & $49(51.04)$ & $45(70.31)$ & 0.015 \\
Remote symptomatic & $14(8.75)$ & 9 & 5 & 0.731 \\
Structural malformation* & $3(1.9)$ & 2 & 1 & 0.811 \\
Idiopathic & $24(15.0)$ & 0 & 8 & 0.469 \\
Epilepsy syndrome, temporal lobe epilepsy & $1(0.6)$ & $20(28.98)$ & $4(6.25)$ & 0.011 \\
FUS & $24(15.0)$ & & 16 & \\
\hline
\end{tabular}

Values are presented as number (\%).

FUS, first unprovoked afebrile seizure.

${ }^{*} A$, enlarged cisterna magna; $B$, encephalomalacia with gliosis; C, arnold chiari malformation.

in group II an additional information was retrieved by EEG, which affected the management plan in only 12 (idiopathic epilepsy and epilepsy syndrome) out of 75 patients ( $p=0.902$ ). Thus, performing MRI brain first subsequently followed by EEG gave a better yield in terms of etiological diagnosis and helped deciding the specific treatment in our study population. Additionally, the median time taken for first investigation as per group allocation (i.e., EEG and neuroimaging) and the median number of hospital visits were also significantly less in group I compared to group II. On further analysis for determining the etiological diagnosis among the two groups (Table 3), inflammatory granuloma (NCC and tuberculoma) ( $p=0.732$ ) was found to be the most common cause of first seizure followed by idiopathic epilepsy ( $p=0.9117)$. Fourteen cases in which no etiologic diagnosis could be made were labeled as FUS.

Among all the patients, irrespective of the group (Table 4), inflammatory granuloma (NCC and tuberculoma) was again the most common etiology found in 94 patients (58.75\%) followed by first unprovoked seizure and idiopathic epilepsy, each found in 24 patients (15\%). Structural malformation and epilepsy syndrome (temporal lobe epilepsy) were the least common causes seen in three (1.9\%) and one patient $(0.6 \%)$, respectively. Also, a statistically significant difference was found in the type of seizure with generalized seizure being more common as compared to focal seizure in patients with first afebrile unprovoked seizure as an etiological diagnosis $(p<0.05)$. However, children with focal seizure had more chances of inflammatory granuloma $(70.3 \%)$ in comparison to those with generalized seizure (51.04\%).

\section{Discussion}

One hundred and seventy subjects with first unprovoked seizure were enrolled in our study. An abnormal MRI was seen in $70.6 \%$ patients in initial MRI group and an abnormal EEG in $61.2 \%$ patients in initial EEG group. Inflammatory granuloma (NCC and tuberculoma) ( $p=0.732$ ) was found to be the most common cause of first seizure followed by idiopathic epilepsy ( $p=0.9117$ ). Initial EEG evaluation shows presence of epileptiform discharges in $18-56 \%$ of children with new onset seizure and no abnormal finding in $15 \%$ of them. ${ }^{14}$ Findings from multiple studies on first unprovoked seizures support similar results. In a study done by Shinnar et al. ${ }^{15} 42 \%$ patients had an abnormal EEG. Moreover, Alehan et al. ${ }^{16}$ reported an abnormal EEG in $56 \%$ patients and King et al. ${ }^{17}$ reported an abnormal EEG in 59\% patients younger 
than 16 years. Our results are comparable with most of these studies. For neuroimaging, results reported by Saini and Baghel ${ }^{1}$ are in concordance with our study where $66.7 \%$ patients of new onset afebrile seizure had an abnormal neuroimaging. In their study, CT head was done in all the patients and few patients underwent additional MRI brain. Positivity rate of CT scan in children for first onset afebrile seizure varies between $21.0 \%$ to $70 \%$ depending upon the study population. ${ }^{18-21}$ Selection bias for cases for choosing CT scan seems to be the plausible explanation for this wide range of positivity rate.

As far as etiology is concerned, focal structural lesion like NCC and tuberculoma remains the leading causes of first unprovoked seizure in children in the developing countries including India. ${ }^{22}$ Results of our study also showed a considerably higher proportion of inflammatory granuloma as an etiological diagnosis of FAS seen in $58.75 \%$ of patients. The study conducted by Mathur et al. ${ }^{23}$ revealed similar results where 17 out of 32 patients with an abnormal neuroimaging had inflammatory granuloma (nine NCC and eight tuberculoma). Saini and Baghel ${ }^{1}$ also found inflammatory granuloma (20 NCC and 13 tuberculoma) as the most common cause in $44 \%$ of patients of new onset afebrile seizure. Another recent study by Chandrakanta et al. ${ }^{24}$ found that $70 \%$ of patients with abnormal neuroimaging had inflammatory granuloma. The current study revealed a very high yield of neuroimaging (70.6\%) as compared to EEG for determining the etiological diagnosis in patients with FAS. Similar results have been reported by various other studies which recommend that a neuroimaging should be done first in patients with FAS taking into consideration the high prevalence of NCC and tuberculosis in developing countries like India. ${ }^{1,23,24}$ Few studies conducted in the West have also revealed similar results. Findings from a study by Shinnar et al. ${ }^{25}$ reveal the yield of neuroimaging in first unprovoked seizures to be very high, but with a low probability of detecting an abnormality requiring immediate medical or surgical intervention. King et al. ${ }^{17}$ also concluded that MRI is indicated in all FAS cases except idiopathic generalized epilepsies or benign rolandic epilepsy as it significantly contributes in diagnosis.

Strengths of the study were, firstly, a thorough follow up of the patients, which was ensured to avoid any bias due to a review of diagnosis after the investigations. Secondly, EEG and MRI brain reporting was done by qualified pediatric neurologists and neuroradiologists, respectively, removing the probability of a false diagnosis. There were a few limitations of this study, the primary one being very few children with an epilepsy syndrome and the other being a delay in getting neuroimaging done for the study participants. A high proportion of children with SSECTL suggests that yield may be different in settings where SSECTL are uncommon.

This study adds to the current knowledge of etiological diagnosis and the yield of diagnostic modalities for the evaluation and treatment of FAS. The results support the precedence of neuroimaging to EEG for evaluation of FAS considering the high incidence of inflammatory granuloma in India, which contrast with the western world guidelines. Additional studies from other centers in India and other developing countries may provide additional information on this topic. The results of our study done in neurologically normal children with FAS showed a high diagnostic yield with an initial MRI. In the light of these results, we recommend that guidelines for evaluation of FAS in children should consider incorporating MRI as the initial investigation, especially in the developing countries.

\section{Conflict of Interest}

The authors declare that they have no conflicts of interest.

\section{References}

1. Saini $N$, Baghel A. Neuroimaging abnormalities in children with first afebrile seizure. J Dent Med Sci 2013;5:21-4.

2. Banerjee PN, Filippi D, Allen Hauser W. The descriptive epidemiology of epilepsy-a review. Epilepsy Res 2009;85:31-45.

3. Sridharan R, Murthy BN. Prevalence and pattern of epilepsy in India. Epilepsia 1999;40:631-6.

4. Arora NK, Nair MKC, Gulati $S$, et al. Neurodevelopmental disorders in children aged 2-9 years: population-based burden estimates across five regions in India. PLoS Med 2018;15:e1002615.

5. Pal DK. Epilepsy control in the 21st century: leave no child behind. Epilepsia 2003;44:273-5.

6. Berg AT, Shinnar $S$. The risk of seizure recurrence following a first unprovoked seizure: a quantitative review. Neurology 1991;41:965-72.

7. Neuroimaging Comission ILAE. ILAE neuroimaging commission recommendations for neuroimaging of patients with epilepsy. Epilepsia 1997; 38 Suppl 10:1-2.

8. Hirtz D, Ashwal S, Berg A, et al. Practice parameter: evaluating a first nonfebrile seizure in children: report of the quality standards subcommittee of the American Academy of Neurology, The Child Neurology Society, and The American Epilepsy Society. Neurology 2000;55:616-23.

9. Expert Committee on Pediatric Epilepsy, Indian Academy of Pediatrics. Guidelines for diagnosis and management of childhood epilepsy. Indian Pediatr 2009;46:681-98.

10. Guidelines for management of epilepsy in India. 18th International Epilepsy Congress Trust; 2018 Oct 1; Gurgaon. Gurgaon: Indian Epilepsy Association; 2008. 28-31 p. 
11. Gaillard WD, Chiron C, Cross JH, et al. Guidelines for imaging infants and children with recent-onset epilepsy. Epilepsia 2009;50:2147-53.

12. Gulati S, Kaushik JS. How I treat a first single seizure in a child. Ann Indian Acad Neurol 2016;19:29-36.

13. Rajshekhar V, Joshi DD, Doanh NQ, van De N, Xiaonong Z. Taenia solium taeniosis/cysticercosis in Asia: epidemiology, impact and issues. Acta Trop 2003;87:53-60.

14. Wirrell EC. Prognostic significance of interictal epileptiform discharges in newly diagnosed seizure disorders. J Clin Neurophysiol 2010;27: 239-48.

15. Shinnar S, Kang H, Berg AT, Goldensohn ES, Hauser WA, Moshé SL. EEG abnormalities in children with a first unprovoked seizure. Epilepsia 1994;35:471-6.

16. Alehan FK, Morton LD, Pellock JM. Utility of electroencephalography in the pediatric emergency department. J Child Neurol 2001;16:484-7.

17. King MA, Newton MR, Jackson GD, et al. Epileptology of the first-seizure presentation: a clinical, electroencephalographic, and magnetic resonance imaging study of 300 consecutive patients. Lancet 1998;352: 1007-11.

18. Gibbs J, Appleton R, Carty H, Beirne M, Acomb BA. Focal electroencephalographic abnormalities and computerised tomography findings in children with seizures. J Neurol Neurosurg Psychiatry 1993;56:369-71.

19. Ladurner $G$, Fritsch $G$, Sager WD, Iliff $L D$, Lechner $H$. Computer tomography in children with epilepsy. Eur Neurol 1980;19:180-4.

20. Bachman DS, Hodges FJ, Freeman JM. Computerized axial tomography in chronic seizure disorders of childhood. Pediatrics 1976;58:828-32.

21. Al-Sulaiman AA, Ismail HM. Clinical pattern of newly-diagnosed seizures in Saudi Arabia: a prospective study of 263 children. Childs Nerv Syst 1999;15:468-71.

22. Sahu PS, Seepana J, Padela S, et al. Neurocysticercosis in children presenting with afebrile seizure: clinical profile, imaging and serodiagnosis. Rev Inst Med Trop Sao Paulo 2014;56:253-8.

23. Mathur $S$, Southern $K$, Sharma M. Significant findings on cranial CT scan after a first unprovoked seizure in children from North India. J Trop Pediatr 2007;53:428-30.

24. Chandrakanta, Verma M, Kumar R, Koonwar S, Gupta S, Parihar A. Role of neuroimaging in children with first unprovoked seizure: a prospective observational study. Eur J Pediatr Neurol 2017;21:e100.

25. Shinnar S, O'Dell C, Mitnick R, Berg AT, Moshe SL. Neuroimaging abnormalities in children with an apparent first unprovoked seizure. Epilepsy Res 2001;43:261-9. 